\title{
Transfusion support of autoimmune hemolytic anemia: how could the blood group genotyping help?
}

\author{
HANANE EL KENZ, ANDRÉ EFIRA, PHU QUOC LE, CLAIRE THIRY, JOSEPH VALSAMIS, \\ MARIE-AGNÈS AZERAD, and FRANCIS CORAZZA
}

BRUSSELS, BELGIUM

\begin{abstract}
Conventional pretransfusion testing based on hemagglutination assays can be challenging for patients with autoimmune hemolytic anemia (AIHA) because of the presence of auto-antibodies. It has been suggested that deoxyribonucleic acid-based methods could be more efficient in the selection of antigen-matched red blood cell units in those settings. Because of the high risk of alloimmunization of these patients and the labor-intensive nature of adsorption techniques, we decided to evaluate the feasibility of selecting antigen-matched units on the basis of RBC genotyping. We included in our routine RBC genotyping program samples from 7 patients with AlHA presenting a strongly positive direct antiglobulin test. This made the routine compatibility tests difficult. Most patients had previously received transfusions because of warm AlHA. Matched donor units were selected according to the genotype. For all but 1 patient, blood group genotyping could be done on time to allow antigen-matched transfusion. Four patients received antigen-matched red blood cell units based on RBC genotyping and for 1 patient the fact that no matched units were available led us to postpone the transfusion. After each transfusion, the recovery was recorded and considered satisfactory for all transfused patients. (Translational Research 2014;163:36-42)
\end{abstract}

\begin{abstract}
Abbreviations: AlHA = autoimmune hemolytic anemia; RBCs = red blood cells; DAT = direct antiglobulin test; DNA = deoxyribonucleic acid; EDTA = ethylenediaminetetraacetic acid; G6PD = glucose-6-phosphate dehydrogenase; INR = International normalized ratio; LISS-IAT $=$ low ionic strength solution-indirect antiglobulin
\end{abstract}

A utoimmune hemolytic anemia (AIHA) is an uncommon disease characterized by an increased destruction of red blood cells (RBCs) mediated by autoantibodies directed against autologous RBCs. AIHA can be classified into different types including warm AIHA, cold agglutinin syndrome, paroxysmal cold hemoglobinuria, mixed-type AIHA, and drug-induced immune hemolytic anemia.

From the Department of Transfusion, Hospital Blood Bank, CHU-Brugmann and Queen Fabiola Children University Hospital, Brussels, Belgium; Department of Hematology, CHU-Brugmann, Brussels, Belgium; Department of Hematology, Queen Fabiola University Children Hospital, Brussels, Belgium; Laboratory of Clinical Biology, CHU-Brugmann, Brussels, Belgium; Laboratory of Immunology, CHU-Brugmann, Brussels, Belgium.

Conflict of interest: None.

H. E. Kenz is a recipient of a grant from the Brugmann Foundation.
Some patients with AIHA may require blood transfusion, according to the severity of anemia and their clinical status. It is generally thought that when the incompatibility is due to the presence of an autoantibody alone, the survival of transfused RBCs is similar to that of a patient's RBCs. However, classical pretransfusion hemagglutination assays are often difficult to interpret for patients with AIHA because of the interference

Submitted for publication July 15, 2013; revision submitted September 16, 2013; accepted for publication September 17, 2013.

Reprint requests: Hanane El Kenz, Pharm D, Department of Transfusion, CHU-Brugmann and Queen Fabiola Children University Hospital, 4 Place Van Gehuchten, B-1020 Brussels, Belgium; e-mail: hanane.elkenz@chu-brugmann.be.

$1931-5244 / \$$ - see front matter

(C) 2014 Mosby, Inc. All rights reserved.

http://dx.doi.org/10.1016/j.trs1.2013.09.007 


\section{AT A GLANCE COMMENTARY}

El Kenz $\mathrm{H}$ et al.

\section{Background}

Patients with autoimmune hemolytic anemia (AIHA) are at high risk to develop a hemolytic transfusion reaction due to an alloantibody. The rate of alloimmunization in these patients is often higher than in other multitransfused patients and, because of autoantibodies, traditional compatibility tests can be challenging or impossible. We, thus, started to determine blood group genotype of patients with AIHA and we report its application and feasibility.

\section{Translational Significance}

In our experience, RBC genotyping techniques for patients with AIHA allow selecting antigenmatched units without laborious, costly, lengthy, and sample consuming adsorption procedures. This application is particularly useful for pediatric patients with small blood samples.

by autoantibodies that can be panreactive. It is essential to exclude these autoantibodies because they can also mask the detection of alloantibodies that can lead to a harmful immuno-hemolytic reaction.

Additionally, the rate of alloimmunization is higher in patients with warm AIHA than in other multitransfused patients. ${ }^{1}$ In fact, several studies report that $12 \%$ to $40 \%$ of patients with warm AIHA have significant alloantibodies..$^{2-8}$ The risk of underlying alloantibodies has prompted transfusion laboratories to provide specialized compatibility testing procedures that allow for the detection of alloantibodies in patients with masking autoantibodies. A variety of methods have been described for the selection of RBCs for patients with autoantibodies. Selecting RBCs for transfusion by providing "least incompatible units" or by testing a patient's diluted serum against a panel of RBCs to detect a more strongly reacting alloantibody are deemed to be unacceptable procedures. ${ }^{1}$ For patients with warm AIHA, the most effective compatibility testing rely on autoadsorption techniques that consist in incubating patient's RBCs at $37^{\circ} \mathrm{C}$ with his or her own serum to remove the autoantibody and to allow detection of clinically significant alloantibodies. In this technique, some of the antibody is also eluted from the patient RBCs thanks to a treatment with proteolytic enzyme. A less labor-intensive alternative involves an autoadsorption using polyethylene glycol, which negates the need for an ad- ditional enzymatic step. ${ }^{1,9,10}$ This technique, although less labor-intensive, might, however, not be feasible in patients with severe anemia, as the large volume of autologous blood required for autoadsorption procedure is not always available. Moreover, if the patient has already received transfusions in the past, the validity of an autoadsorption is questionable because of the mixture of the patient and donor RBCs. ${ }^{11}$ The most suitable procedure will then consist in adsorption using allogeneic RBCs of different known phenotypes that allows removing the autoantibody from the patient's serum.

In general, adsorption procedures are heavy, lengthy, and sample-consuming. A strong collaboration between the clinician and the blood bank staff is, therefore, essential to evaluate the need and the urgency of the transfusion and to guide the selection of appropriate compatibility test procedures in a way that will provide RBC units as safely and timely as possible.

Depending on the urgency of the transfusion need, the results may not be available prior to the transfusion of the first RBC unit but remain useful for further transfusions. For these reasons, the determination of the most complete phenotype (including RH1, RH2, RH3, RH4, RH5, KEL1, FY1, FY2, JK1, JK2, MNS1, MNS2, MNS3, and MNS4 antigens) coupled with the delivery of prophylactic antigen-matched donor blood has been proposed as an alternative method to the adsorption techniques ${ }^{12}$ for patients with warm AIHA. This approach is only applicable when the patient does not have a strongly positive direct antiglobulin test (DAT) or has not been transfused. ${ }^{12}$ In cases of patients with recent transfusions or strongly positive DATs, there are no effective serologic alternatives to adsorption procedures.

Although hemagglutination remains the gold standard method in immunohematology reference laboratories, molecular immunohematology is increasingly used as a valuable method to provide appropriate transfusion support, to reduce the risk of transfusion reactions, and to prevent alloimmunization in multitransfused patients such as those with sickle cell disease patients or thalassemia. ${ }^{13,14}$ Blood group genotyping is also used to detect variants when hemagglutination tests show a weak or altered antigen expression, especially in a context of pregnancy or previous transfusion. ${ }^{15,16}$

Therefore, we have considered the option to apply blood group genotyping using a highthroughput deoxyribonucleic acid (DNA) assay rather than extended RBC phenotyping to manage the transfusion support of patients with AIHA who have a high risk of alloimmunization and are likely to be multitransfused. 


\section{METHODS}

Patient selection. The study protocol was carried out according to the principles of the Declaration of Helsinki. During a period of 1 year, samples from patients with AIHA $(n=7)$ were included in the routine blood group genotyping program. This program was adapted to include EDTA blood samples collected from patients with warm autoantibodies as quickly as possible based on the availability of qualified laboratory staff and on the time of sample receipt. For 1 patient out of 7 , it was not possible to wait for the genotyping before transfusion of the first blood unit, and this was due to poorly supported anemia. When the sample had a strongly positive DAT with anti-IgG and a panagglutinin in the plasma making antibody screens and cross-matching difficult, genomic DNA was extracted from an aliquot of $200 \mu \mathrm{L}$ EDTA whole blood sample using the QIAamp DNA Blood Minikit (Qiagen). The DNA sample was included in the routine batch of samples waiting for genotyping.

Genotyping method. Molecular analyses were performed using the BioArray HEA BeadChip (Immucor, Warren, NJ). Polymerase chain reaction amplification was performed in a Veriti thermal cycler (Applied Biosystems) with a set of primers selected by the manufacturer to generate the amplicons with the polymorphic sites of interest. After an elongation step, polymerase chain reaction products were incubated on BeadChip (Immucor) carriers. The fluorescence pattern was acquired and analyzed by the proprietary BASIS software to generate phenotype and genotype reports.

Donor units matched to the patient's phenotype were selected according to RH1, RH2, RH3, RH4, RH5, KEL1, FY1, FY2, JK1, JK2, MNS1, MNS2, MNS3, and MNS4 antigens. However, the routinely used BeadChip (Immucor) allows determination of minor polymorphisms, in addition to these major group systems. When necessary, 2 other panels are available for variants for the Rh blood group system. This approach allows reliable genotyping of almost all patients except very rare unknown variants requiring gene sequencing. ${ }^{15}$

All routine tests, such as low-ionic-strength solutionindirect antiglobulin (LISS-IAT) screen and antibody identification panel, were performed by hemagglutination gel test according to the manufacturer instructions (BioRad). DAT was performed with the gel test using polyspecific anti-IgG and anti-C3 reagents and the eluate with a commercial test kit (BioRad).

\section{RESULTS}

We present here a description of 7 patients with AIHA for whom we have introduced blood group genotyping in our transfusion management strategy.
Patient no. 1. The first patient was a 70-year-old woman who developed warm AIHA secondary to chronic lymphocytic leukemia and had previously required transfusion of 13 RBCs units in 7 episodes. She was admitted to our emergency department with dyspnea and pyrexia. Hemoglobin ( $\mathrm{Hb})$ concentration was $6.6 \mathrm{~g} / \mathrm{dL}$. The DAT was strongly positive with autoantibodies that impeded all routine compatibility tests including the complete phenotype. The LISS-IAT was positive with a $3+$ reactivity. Six hours later, she was transfused with 2 selected RBC units matched to her phenotype that is RH:1,2,-3,4,5; KEL:-1,2; JK:1,2; FY:-1,2; MNS:1,-2,3,4.

The first transfusion episode was followed by 6 additional episodes (see Table I). As for the first transfusion episode, the DAT and LISS-IAT remained positive with a $2+$ to $3+$ reactivity. All donor RBC units administered were matched to her genotype avoiding any additional auto- or alloadsorption procedures before transfusion. The latter were, however, programed after the first, the third, and the sixth transfusion episode and revealed no new alloantibody. No transfusion reaction was reported, and the RBC recovery was evaluated after each transfusion.

Patient no. 2. The second patient, an 8-year-old girl, was admitted to the emergency department for an AIHA in the context of Aicardi-Goutieres syndrome with an $\mathrm{Hb}$ concentration of $7.2 \mathrm{~g} / \mathrm{dL}$. Three years ago, she had an episode of AIHA that required transfusion of $2 \mathrm{RBC}$ units. Because of the patient pathology and her transfusion history, she was considered at risk for further transfusions.

All routine compatibility tests, including the complete serologic phenotype, were confusing due to a strongly positive DAT with anti-IgG and anti-C3d and the presence in her plasma of a pan-agglutinin. We performed RBC genotyping that helped us to preselect an antigen-matched RBC unit ordered to our Blood Transfusion Service. This antigen-matched unit (RH:1,2,-3,-4,5; KEL:-1,2; JK:1,2; FY:-1,2; MNS:1,$2,-3,4)$ was transfused during her stay when her $\mathrm{Hb}$ fell to $4.9 \mathrm{~g} / \mathrm{dL}$. Post-transfusion $\mathrm{Hb}$ concentration reached $9.4 \mathrm{~g} / \mathrm{dL}$ (see Table I) without any transfusion reaction. The adsorption procedure performed 1 week after the transfusion did not reveal the presence of any alloantibody.

Patient no. 3. The third patient, an 83-year-old woman, was admitted to our emergency department with chest pain for 4 days and pain in her right hypochondrium. She was under anticoagulant treatment since a previous episode of venous thromboembolism. International normalized ratio increased at admission revealed an overdose in acenocoumarol. She was pale and icteric, and her $\mathrm{Hb}$ concentration was $7.7 \mathrm{~g} / \mathrm{dL}$, haptoglobin 
Table I. Transfusion episodes

\begin{tabular}{|c|c|c|c|c|c|}
\hline $\begin{array}{l}\text { Transfusion } \\
\text { episodes }\end{array}$ & $\begin{array}{l}\text { Number } \\
\text { of units }\end{array}$ & Transfused RBCs & $\begin{array}{l}\mathrm{Hb}(\mathrm{g} / \mathrm{dL}) \text { before } \\
\text { transfusion }\end{array}$ & $\begin{array}{l}\mathrm{Hb}(\mathrm{g} / \mathrm{dL}) \text { after } \\
\text { transfusion }\end{array}$ & $\begin{array}{l}\text { Alloantibodies detected } \\
\text { at least } 1 \text { week } \\
\text { post-transfusion }\end{array}$ \\
\hline \multicolumn{6}{|l|}{ Patient no.1 } \\
\hline 1 & 2 & RH:-3; KEL:-1; FY:-1; MNS:-2 & 5.1 & 7.7 & None \\
\hline 2 & 2 & RH:-3; KEL:-1; FY:-1; MNS:-2 & 4.8 & 7.8 & \\
\hline 3 & 2 & RH:-3; KEL:-1; FY:-1; MNS:-2 & 6.8 & 8.8 & None \\
\hline 4 & 2 & RH:-3; KEL:-1; FY:-1; MNS:-2 & 5.2 & 7.3 & \\
\hline 5 & 1 & RH:-3; KEL:-1; FY:-1; MNS:-2 & 5.4 & 6.5 & \\
\hline 6 & 2 & RH:-3; KEL:-1; FY:-1; MNS:-2 & 6.9 & 10.1 & None \\
\hline 7 & 2 & RH:-3; KEL:-1; FY:-1; MNS:-2 & 5.8 & 7.2 & \\
\hline \multicolumn{6}{|c|}{ Patient no. 2} \\
\hline 1 & 1 & RH:-3;-4; KEL:-1; FY:-1; MNS:-2,-3 & 4.9 & 9.4 & None \\
\hline \multicolumn{6}{|c|}{ Patient no. 3} \\
\hline 1 & 1 & RH:-2,-5; JK:-1; FY:-2; MNS:-2 & 6.9 & 7.6 & None \\
\hline 2 & 2 & RH:-2,-5; JK:-1; FY:-2; MNS:-2 & 7.1 & $5.8^{*}$ & \\
\hline 3 & 9 & Transfusion of not matched units & $4.9^{\dagger}$ & 8.1 & \\
\hline 4 & 4 & $\mathrm{RH}:-2,-5 ; \mathrm{JK}:-1 ; \mathrm{FY}:-2 ; \mathrm{MNS}:-2$ & 7.3 & 12.2 & None \\
\hline \multicolumn{6}{|c|}{ Patient no. 4} \\
\hline 1 & 1 & Transfusion of not matched unit & 6 & 9.3 & \\
\hline 2 & 1 & $\mathrm{RH}:-3,-4 ; \mathrm{KEL}:-1 ; \mathrm{MNS}:-3$ & 3.1 & 8.0 & \\
\hline 3 & 1 & $\mathrm{RH}:-3,-4 ; \mathrm{KEL}:-1 ; \mathrm{MNS}:-3$ & 4.9 & 7.7 & \\
\hline
\end{tabular}

Abbreviations: $\mathrm{Hb}$, hemoglobin; RBC, red blood cell.

${ }^{*} \mathrm{Hb}$ concentrations remaining low because of gastrointestinal bleeding.

${ }^{\mathrm{t}} \mathrm{Hb}$ concentrations during the episode of acute gastrointestinal bleeding.

was indetectable, and the DAT was strongly positive with IgG auto-antibodies that precluded all routine compatibility tests including the complete phenotype. The LISS-IAT was positive with 3 + reactivity.

The decision to postpone transfusion was made by the emergency physician, and the patient was transferred to the hematology department. The following RBC genotype was identified: RH:1,-2,3,4,-5; KEL:1,2; JK:-1,2; FY:1,-2; MNS:1,-2,3,4. Because of heart failure, the hematologist prescribes the transfusion of 1 matched RBC unit (see Table I). During her stay, her $\mathrm{Hb}$ concentration continued to decline, the esophago-gastroduodenoscopy revealed the presence of duodenal ulcers causing a gastrointestinal bleeding, and two additional RBC units matched were transfused. Two days later, she was transferred to the intensive care unit for an acute gastrointestinal bleeding requiring the massive transfusion of 9 RBCs units, 8 fresh frozen plasmas, and 2 platelet concentrates. However, during this acute transfusion episode, we could not match with her complete phenotype for the units administered. After being stabilized, 4 other RBC units were finally transfused with correctly matched units in another transfusion episode. Adsorption procedures were performed before the first and after the last transfusion episode, and no alloantibodies could be been detected.

Patient no. 4. An almost 3-year-old girl was admitted to the emergency department for severe $S$. pneumoniae infection, associated with acute renal failure, thrombocytopenia, and AIHA. We found a strongly positive DAT with anti-IgG and anti-C3d and the presence in her plasma of a pan-agglutinin impeding our routine compatibility tests. She had no history of previous transfusion. At admission, her $\mathrm{Hb}$ concentration was $6.0 \mathrm{~g} / \mathrm{dL}$. She was transferred to the intensive care unit where she received a first nonmatched RBC unit. Because of the high risk of further transfusions and the impossibility to conduct any adsorption studies because of the lack of sufficient serum samples, we decided with the physician to perform a RBC genotyping.

During her hospitalization, 2 additional preselected antigen-matched blood units RH:1,2,-3,-4,5; KEL:-1,2; JK:1,2; FY:1,2; MNS:1,2,-3,4 were administered (see Table I).

Patient no. 5. An 80-year-old man developed AIHA secondary to myelodysplastic syndrome and had a history of transfusion episodes. During this period, DAT was strongly positive with anti-IgG and anti-C3d, and the autoantibodies impeded all routine compatibility tests including the complete phenotype. The LISS-IAT was positive with $2+$ / $3+$ reactivity.

Adsorption studies of his samples were performed prior to each transfusion. A significant alloantibody known to cause serious hemolytic transfusion reactions (anti-Jka) was discovered after 1 of these adsorption procedures. During the last hemolytic episode, four 
Jka- RBCs units were transfused. The alloadsorption procedure was laborious and was not available before the transfusion of the first unit.

This patient was regularly followed up in the hemato-oncology clinic and was included in the RBC genotyping protocol. Genotyping revealed a common Caucasian RBC phenotype, which was easily found in our donor population. In case of the for additional $\mathrm{RBC}$, further units will be immediately selected according to his genotype without performing any other adsorption procedure.

Patient no. 6. The sixth patient, a 38-year-old man, presented 1 year before with a primary AIHA for which he had required an emergency transfusion of 2 RBC units. He was regularly followed at the outpatient hematology clinic. He had a consistent strongly positive DAT with anti-IgG, a $2+$ reactivity positive LISS-IAT and an $\mathrm{Hb}$ level at the lowest limit of normal range. This patient remained at risk for a new hemolytic episode as in most patients with primary AIHA. ${ }^{17}$ Given this context, we conducted genotyping of blood groups to subsequently allow fast selection of most suitable units of RBC.

Patient no. 7. An 11-year-old African boy was presented with a complex hemolytic anemia caused by both a known G6PD deficiency and a recurrent primary AIHA. He was admitted to the emergency department for abdominal pain. At the admission, he had an $\mathrm{Hb}$ concentration of $5.2 \mathrm{~g} / \mathrm{dL}$, haptoglobin $<3 \mathrm{mg} / \mathrm{dL}$, a strongly positive DAT with anti-IgG, and anti-C3d and a panagglutinin impeding our routine compatibility tests. His important transfusion history (10 RBCs transfused in the past) led us to immediately start autoadsorption procedure, which failed because we could not get enough blood for all tests.

We, therefore, decided to perform a blood group genotyping before any further transfusion. His genotype was determined as RH:1,-2,-3,4,5; KEL:-1,2; JK:1,-2; FY:-1,-2; MNS:-1,2,-3,4. Matched blood was not immediately available due to this rare phenotype in our population of mostly Caucasian blood donor. We, therefore, postponed the transfusion, in agreement with the pediatrician, since the patient was clinically stable. Corticosteroids started at day 0 induced a rapid increase in hemoglobin $(3 \mathrm{~g} / \mathrm{dL}$ in 10 days). Autoadsorption procedures were carried out as soon as his hemoglobin was high enough to collect the sample of blood required, and it revealed the presence of an anti-RH5. The molecular analysis of the most frequent mutations responsible for RH5 variants ruled out the presence of a partial RH5 antigen that would induce an alloimmunization. We, thus, considered the anti-RH5 as an autoantibody.

\section{DISCUSSION}

Patients with AIHA are at high risk to develop a hemolytic transfusion reaction because of an alloantibody. The rate of alloimmunization in these patients is even higher than in other multitransfused patients such as those with sickle cell disease or thalassemia. ${ }^{1}$ Moreover, traditional compatibility tests can be challenging or impossible because of the presence of autoantibodies.

For these reasons, as recently recommended,${ }^{14}$ we started to genotype patients with AIHA to determine their blood group antigens, and we here report its application and feasibility for 7 patients. For every patient before each transfusion, the physician was informed of the genotyping procedure for compatibility testing, and he was asked to keep us informed of posttransfusion recovery.

For some patients, genotyping allowed us to avoid systematic labor-intensive autoadsorption procedures for the following episodes of transfusion.

Four patients presenting with AIHA had a hematooncologic pathology.

- For the first patient, who had an important history of transfusions, this transfusion strategy was very beneficial. Indeed, the matched units selected in this way were transfused in at least 6 other transfusion episodes with satisfactory post-transfusion recovery.

- The history of patient no. 5 revealed a large number of previous transfusions that had required the application of multiple laborious autoadsorption procedures, allowing to discover an allo- anti-Jka. RBC genotyping for this patient was an option to facilitate faster and easier selection of compatible units of blood, thanks to the wide availability of units of its phenotype.

- Patient no. 6 presented with primary AIHA and had previously received 2 blood units. He still had a strongly positive DAT (IgG type) even without additional hemolytic episodes. The decision to perform the genotyping as a preventive measure was made with his primary physician.

- For patient no.7 who presented with an Hb concentration of $5.2 \mathrm{~g} / \mathrm{dL}$, we could infer the blood group phenotype despite the small amount of sample. Because of the unavailability of this phenotype in our blood bank, we decided to postpone the transfusion.

Contrary to a widespread assumption, it is important to note that hemato-oncologic patients have a similar risk of alloimmunization as other multitransfused patients. ${ }^{17}$ In addition, increased incidence of AIHA for these patients could be related to the more widespread usage of purine analogs. ${ }^{18}$ Most of these patients receive multiple transfusions, either planned or in an emergency 
setting. As a result, for hemato-oncologic patients presenting with a strongly positive DAT with anti-IgG and panagglutinin in the plasma, $\mathrm{RBC}$ genotyping enables antigen-matched unit selection as soon as the need for a transfusion is known.

In pediatrics, RBC genotyping offers an additional advantage over the auto-adsorptions because of difficulties in collecting sufficient blood volume for adsorption studies.

Indeed, for 2 of the 3 pediatric patients, we could perform genotyping before deciding on the transfusion. This was important given that these patients had been transfused in the past and because of the unavailability of samples for autoadsorptions. For the child who has been transfused without matched RBCs and without autoadsorption testing, we took into account the fact that he had not been previously transfused. For the following transfusions of this patient, the blood group genotype was then available, and we transfused only matched RBCs.

In the study of Shirey et $\mathrm{al}^{12}$ who realized an extended typing of patients with AIHA, the typing could not be achieved (not possible) in $40 \%$ of the cases and autoadsorption tests have been performed to find a potential alloantibody. In contrast to genotyping, extended typing with serology often cannot be conducted for all patients because of the limited availability of certain antisera and prior transfusion history. Although RBC genotyping cannot be performed in all immunohematology laboratories, as it is the case for adsorption techniques, these analyses can be accommodated by reference laboratories, which have a sufficiently high genotyping activity to treat emergency samples without delay.

This procedure requires a strong understanding by the physician of the issues related to red blood cell compatibility testing and of the benefits of RBC genotyping for these patients. Applying such a procedure allows to decrease alloimmunizations and to lower the risk of transfusion reactions. In addition, $\mathrm{RBC}$ genotyping has the potential to decrease the workload and cost associated with complex serologic compatibility testing, especially in patients who are multitransfused.

It is also to be noted that all but 1 patient tested in this study presented Caucasian RBC phenotypes, which led to an easier selection of antigen-matched units. This procedure could become more difficult if we have to match genotype between patients and donors with dissimilar ethnicities. In our institution, most of blood donors are Caucasian and that can lead to difficulties to find matched blood for some patients as illustrated by patient no. 7. For these recipients, we need to maintain our adsorption procedures; however, even in these cases, genotyping enables the selection of fewer panel cells for alloadsorption.
For patients with AIHA, testing for the detection of alloantibodies takes a minimum of 4 to 6 hours, needs large samples, and may not be contributive.

Therefore, RBC genotyping, if available, must be proposed and discussed with the physician who will balance the risk of waiting several hours for matched $\mathrm{RBC}$ units with the risk of hemolytic reactions.

In conclusion, RBC genotyping techniques for patients with AIHA allow selecting antigen-matched units without heavy, costly, lengthy, and sample consuming adsorption procedures. This application is particularly useful for pediatric patients with small blood samples. However, adsorption methods may still be needed when the patient's genotype does not allow antigenmatched units selection. In case of former transfusions, the patient's genotype is still useful for selecting panel cells used in the alloadsorption. Communication between blood bank staff and the hematologist in charge of the patient is essential, a key success factor in the implementation of this procedure.

Although a small number of samples have been tested, we have found that RBC genotyping is highly beneficial for patients with AIHA and makes the selection of compatible blood considerably easier, even in an emergency context. A larger study should, however, be conducted to confirm that genotyping is more efficient than the current serologic procedure to reduce the risk of alloimmunization, delayed hemolytic transfusion reaction, and decreased RBC survival because of an undetected alloantibody.

\section{REFERENCES}

1. Branch DR, Petz LD. Detecting alloantibodies in patients with autoantibodies. Transfusion 1999;39:6-10.

2. Leger RM, Garatty G. Evaluation of methods for detecting alloantibodies underlying warm autoantibodies. Transfusion 1999;39: $11-6$.

3. Branch DR, Petz LD. A new reagent (ZZAP) having multiple applications in immunohematology. Am J Clin Pathol 1982;78: 161-7.

4. Wallhermfechtel MA, Pohl BA, Chaplin H. Alloimmunization in patients with warm autoantibodies: a retrospective study employing three donor alloabsorptions to aid in antibody detection. Transfusion 1984;24:482-5.

5. James P, Rowe GP, Tozzo GG. Elucidation of alloantibodies in autoimmune haemolytic anaemia. Vox Sang 1988;54:167-71.

6. Sokol RJ, Hewitt S, Booker DJ, Morris BM. Patients with red cell autoantibodies: selection of blood for transfusion. Clin Lab Haematol 1988;10:257-64.

7. Laine ML, Beattie KM. Frequency of alloantibodies accompanying autoantibodies. Transfusion 1985;25:545-6.

8. Issitt PD, Combs PR, Bumgarner DJ, Allen J, Kirkland A, Melroy-Carawan H. Studies of antibodies in the sera of patients who have made red cell autoantibodies. Transfusion 1996;36: 481-6.

9. Cheng CK, Wong ML, Lee AW. PEG adsorption of autoantibodies and detection of alloantibodies in warm autoimmune hemolytic anemia. Transfusion 2001;41:13-7. 
10. Cid J, Ortin X, Pinacho A, Parra R, Contreras E, Elies E. Use of polyethylene glycol for performing autologous adsorptions. Transfusion 2005;45:694-7.

11. Laine EP, Leger RM, Arndt PA, Calhoun L, Garatty G, Petz LD. In vitro studies of the impact of transfusion on the detection of alloantibodies after autoadsorption. Transfusion 2000;40:1384-7.

12. Shirey RS, Boyd JS, Parwani AV, Tanz WS, Ness PM, King KE. Prophylactic antigen-matched donor blood for patients with warm autoantibodies: an algorithm for transfusion management. Transfusion 2002; 42:1435-41.

13. Reid ME, Denomme GE. DNA-based Methods in the Immunohematology Reference Laboratory. Transfu Apher Sci 2001;44: $65-72$.
14. Anstee DJ. Red cell genotyping and the future of pretransfusion testing. Blood 2009;114:248-56.

15. Kappler-Gratias S, Peyrard T, Beolet M, et al. Blood group genotyping by high-throughput DNA analysis applied to 356 reagent red blood cell samples. Transfusion 2011;51:36-42.

16. Pham BN, Roussel M, Peyrard T, et al. Anti-D investigations in individuals expressing weak D Type 1 or weak D Type 2: alloor autoantibodies? Transfusion 2011;51:2679-85.

17. Schonewille H, de Vries RR, Brand A. Alloimmune response after additional red blood cell antigen challenge in immunized hematooncology patients. Transfusion 2009;49:453-7.

18. Garratty G. Drug-induced immune hemolytic anemia. Hematology Am Soc Hematol Educ Program 2009;1:73-9. 